Review

\title{
microRNA biomarkers in colorectal cancer liver metastasis
}

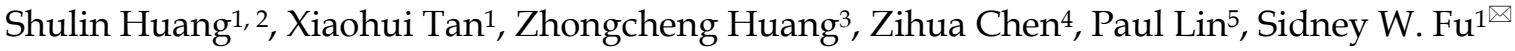 \\ 1. Department of Medicine (Division of Genomic Medicine), The George Washington University School of Medicine and Health Sciences, Washington, DC \\ 2. Department of Breast and Thyroid Surgery, Hunan Provincial People's Hospital (The First Affiliated Hospital of Hunan Normal University), Changsha, \\ Hunan, China \\ 3. Department of Colorectal and Anal Surgery, Hunan Provincial People's Hospital (The First Affiliated Hospital of Hunan Normal University), Changsha, \\ Hunan, China \\ 4. Hepatobiliary and enteric Surgery Research Center/Department of Gastrointestinal Surgery, Xiangya Hospital, Central South University, Changsha, \\ Hunan, China \\ 5. Department of Surgery, The George Washington University School of Medicine and Health Sciences, Washington, DC
}

$\square$ Corresponding author: Sidney W. Fu, M.D., Ph.D. Department of Medicine, Division of Genomic Medicine, The George Washington University School of Medicine and Health Sciences, 2300 Eye Street, N.W. Ross Hall 402C, Washington, DC 20037 Email: sfu@gwu.edu Tel: 202-994-4767

( ) Ivyspring International Publisher. This is an open access article distributed under the terms of the Creative Commons Attribution (CC BY-NC) license (https://creativecommons.org/licenses/by-nc/4.0/). See http://ivyspring.com/terms for full terms and conditions.

Received: 2018.07.17; Accepted: 2018.08.20; Published: 2018.10.06

\begin{abstract}
Liver metastasis is a primary factor of prognosis and long-term survival for patients diagnosed with colorectal cancer (CRC). Colorectal cancer liver metastasis (CRCLM), is a complex biological process involving multiple factors and steps, and its mechanisms are yet to be discovered. In recent years, small noncoding RNAs, especially microRNAs (miRNAs) have been proven to play an important role in tumorigenesis, progression and metastasis in a variety of cancers, including CRC. Increasing evidence suggests that miRNAs, including those from exosomes secreted by tumor cells in circulation, could be used as promising biomarkers in early cancer detection, treatment, and prognosis. In this review, we focus on the functional roles and clinical applications of miRNAs, especially those from circulating exosomes secreted by tumor cells related to CRCLM.
\end{abstract}

Key words: Colorectal cancer (CRC), liver metastasis, miRNAs, exosome

\section{Introduction}

Colorectal cancer (CRC) is one of the most commonly diagnosed cancers worldwide $[1,2]$. CRC is typically treated by surgical resection combined with modern chemo- and radiation- therapies, with a cure rate of $\sim 20 \%$ [3]. About $70-80 \%$ of CRC patients will develop recurrence and/or metastasis, and more than $50 \%$ of the metastatic sites are in the liver $[3,4]$. Colorectal cancer liver metastasis (CRCLM) is the leading cause of deaths for CRC [5]. Therefore, one of the main research focuses is on elucidating the mechanisms of CRCLM, and identifying potential biomarkers for both prediction and management of CRCLM [6]. Better understanding of the biological process of CRCLM will facilitate the development of early diagnostic and therapeutic methods. In recent years, small non-coding RNAs (sncRNAs) have been shown to play a key role in the regulation of gene expression and are involved in tumor initiation and development. These sncRNAs include small interfering RNAs (siRNAs), microRNAs (miRNAs), PIWI-interacting RNAs (piRNAs), etc., of which miRNAs are studied most extensively and in-depth [7, 8]. In this review, we will focus on the roles and clinical applications of miRNAs in CRCLM, including those in circulating exosomes secreted by tumor cells.

miRNAs are endogenous non-coding RNAs of 20-25 nucleotides that regulate gene expression at the post-transcriptional level through interaction with $3^{\prime}$-untranslated regions (3'UTRs) of the target messenger RNAs (mRNAs) [9]. Each miRNA may target a number of mRNAs, and each mRNA can be regulated by multiple miRNAs. Studies have shown that miRNAs play an important regulatory role in all aspects of CRCLM, including angiogenesis, tumor 
invasion, epithelial-to-mesenchymal transition (EMT), and stemness of cancer cells [10]. Recently miRNAs have been used in the diagnosis and prognosis of cancer and other diseases [11]. However, metastasis of cancers, such as CRC, is not only challenging to predict, but also has limited and ineffective treatment options. Therefore, it is crucial to discover and validate novel biomarkers that can predict CRCLM in order to improve the survival rate of patients. A number of publications demonstrated that miRNAs, either oncogenic miRNAs (OncomiRs) or tumor suppressor miRNAs, are involved in CRCLM (Table 1).

Table 1. A list of miRNAs and their validated targets involving CRCLM

\begin{tabular}{|c|c|c|c|}
\hline miRNAs & Validated targets & Functional pathways & References \\
\hline \multicolumn{4}{|l|}{ OncomiRs } \\
\hline miR-19 & TG2 & metastasis & [12] \\
\hline miR-885-5p & Cpeb2 & EMT & [13] \\
\hline miR-20a-5p & Smad4 & EMT & [14] \\
\hline miR-21 & PPCD4 & Proliferation, invasion & [16] \\
\hline miR-155 & TP53INP1 & Lymph node metastasis & [16] \\
\hline miR-181a & WIF-1 & EMT, metastasis & [17] \\
\hline $\operatorname{miR}-429$ & SOX2 & Apoptosis & [18] \\
\hline \multicolumn{4}{|c|}{ Tumor suppressors } \\
\hline miR-200c & Bmi1, ZEB1, ZEB2 & ERK1/2MAPK/GSK-2 $\beta / \beta$-catenin signaling, fas signaling & [19] \\
\hline $\mathrm{miR}-26 \mathrm{a} / 26 \mathrm{~b}$ & FUT4 & metastasis & [20] \\
\hline miR-30e-5p & ITGA6,ITGB1 & Proliferation, metastasis & [21] \\
\hline $\operatorname{miR}-125$ & HIF-12, VEGF & angiogenesis & [22] \\
\hline miR-127 & BCL6 & differentiation & [22] \\
\hline miR-145 & c-Myc, N-RAS & angiogenesis & [22] \\
\hline miR-194 & BMP1, CDKN1B & Proliferation & [22] \\
\hline miR-199a-30 & HIF-1a, VEGF & Proliferation, motility, invasion & [22] \\
\hline miR-15b & MTSS1, Klotho & Invasion, metastasis & [23] \\
\hline $\operatorname{miR}-30 a-5 p$ & TM4SF1 & EMT & [24] \\
\hline
\end{tabular}

Table 2. A list of miRNAs with their respective clinical applications in CRCLM

\begin{tabular}{|c|c|c|c|c|}
\hline miRNAs & sample & Source of sample & Clinical application/biological function & References \\
\hline miR-29a & Serum & CRCLM & Early detection & {$[26]$} \\
\hline miR-126 & Serum & CRC/CRCLM & Early detection & [27] \\
\hline miR-141 & Serum & CRC/CRCLM & Early detection & [27] \\
\hline miR-21 & Serum & CRC/CRCLM & Early detection & [27] \\
\hline $\operatorname{miR}-29$ & Tissue mouse model) & CRC/CRCLM & Therapeutic target & {$[28]$} \\
\hline miR-622 & Tissue/cell lines & CRC/CRCLM & Therapeutic target & [29] \\
\hline miR-21 & Cell lines/CAM module & CRC/CRCLM & Therapeutic target & {$[30]$} \\
\hline miR-655-3p & Tissue (mouse model) & CRC/CRCLM & Therapeutic target & {$[31]$} \\
\hline miR-424-3p,503,1292 (combine together) & Tissue & CRC/CRCLM & Therapeutic target & [32] \\
\hline let-7i & Tissue/serum & CRC/CRCLM & Prognostic & [33] \\
\hline miR-10b & Tissue/serum & CRC/CRCLM & Prognostic & [34], [38] \\
\hline $\operatorname{miR}-885-5 p$ & Serum & CRC/CRCLM & Prognostic & [33] \\
\hline miR-214 & Tissue & CRC/CRCLM & Prognostic & [35] \\
\hline miR-625 & Tissue & CRC/CRCLM & Prognostic & [36] \\
\hline miR-122 & Tissue & CRC/CRCLM & Prognostic & [37] \\
\hline miR-196b-5p & Tissue & CRC/CRCLM & Prognostic & [39] \\
\hline miR-203 & Serum & CRC/CRCLM & Prognostic & {$[40],[41]$} \\
\hline Exo-miR-19a, 19b, 23a, 92a, 320a, 4437,19a & Serum & CRC/CRCLM & Predict liver metastasis & {$[45]$} \\
\hline Exo-miR-19a & Serum & CRC/CRCLM & Prognostic & {$[45]$} \\
\hline Exo-miR-7,181a-5p,192,194,375 & Serum/cell culture medium & CRC/CRCLM & Inflammation, fibrosis, angiogenesis & {$[48]$} \\
\hline Exo-miR-210 & Tissue & CRC/CRCLM & carcinogenesis, migration & {$[50]$} \\
\hline Exo-miR-193a & Tissue (mouse model) & CRC/CRCLM & Migration & [51] \\
\hline
\end{tabular}




\section{Oncogenic miRNAs (OncomiRs) in CRCLM}

OncomiRs, which act like oncogenes by downregulating tumor suppressors and other regulatory genes, have been found to be either amplified or overexpressed in many types of cancers, including CRC. Cellura et al. reported that miR-19, significantly overexpressed in CRC cells, acts as an oncomiR, leading to altered invasive ability by inhibiting the expression of Transglutaminase-2 (TG2). TG2 is a critical crosslinking enzyme in the extracellular matrix (ECM) and tumor microenvironment (TME) ${ }^{[12]}$ and plays an important role in colorectal cancer metastasis. Another study showed that up-regulation of miR-885-5p had a significant impact on EMT and the overexpression of miR-885-5p significantly induced cell migration, invasion and liver metastasis by targeting cpeb2, which is required for cell cycle progression [13]. miR-20a-5p promotes invasion and metastasis by suppressing Smad4 in CRCLM [14], while miRNA-21 and miRNA-155 were shown to be overexpressed in many malignant solid tumors, including CRC, thoracic tumors and lung cancer [15]. Shibuya et al[16] found that expression of miR-21 and miR-155 is much higher in tumor tissue compared to the adjacent normal tissue in 156 CRC patients, further demonstrating the correlation between miRNA-21 and miRNA-155, along with their respective target genes PDCD4 and TP53INP1. Interestingly, highly expressed miRNA-21 is positively correlated with CRCLM, which is most likely due to the fact that miRNA-21 promotes tumor cell infiltration and metastasis by inhibiting PDCD4, while miR-155 is associated with distant metastasis of lymph nodes. Ji et al. [17] demonstrated that miR-181a promotes tumorigenesis and EMT by inhibiting WIF-1 in CRCLM. miR-429, as an oncomiR, was shown to inhibit apoptosis and induce EMT in CRC by targeting SOX2 [18]. Overexpression of oncomiRs in CRC may promote liver metastasis; therefore, targeting oncomiRs may open a new avenue to prevent CRCLM and improve survival rate of CRC patients.

\section{Tumor suppressor miRNAs in CRCLM}

Tumor suppressor miRNAs function by targeting genes with oncogenic activity. Inactivation or downregulation of tumor suppressor miRNAs was widely reported in many types of cancers including CRC. Bmi1 plays an essential role in Fas-signaling induced stem cell properties in CRC specimens. It was shown that Fas ligand treatment promoted Bmi1 expression by inhibiting miR-200c, a tumor suppressor miRNA [19]. Li et al. [20] discovered that forced expression of miR-26a and miR-26b not only modulates migratory behavior of CRC cells but also inhibits FUT4 expression in CRC cells, resulting in tumor progression. Laudato et al. [21] found that miR-30e-5p inhibits CRCLM by directly targeting both ITG6 and TGF-B1. Another study showed that there are 939 human miRNAs related to CRCLM. Among them, miR-125, miR-127, miR-145, miR-194 and miR-199a-30 act as tumor suppressors and could serve as biomarkers for early diagnosis of CRCLM [22]. Two other recent studies demonstrated that miR-15b inhibits CRCLM by regulating MTSS1 and Klotho [23], while miRNA-30a-5p (miR-30a) regulates cell motility and EMT by directly targeting oncogenic protein TM4SF1 in CRCLM [24]. Downregulation of tumor suppressor miRNAs may lead to CRCLM and detection of these miRNAs may help manage the progression of CRCLM.

\section{miRNA biomarkers CRCLM}

It is crucial to identify and validate novel biomarkers that can predict CRCLM in order to improve patients' survival. Many miRNAs are associated with early diagnosis and staging of CRCLM as discussed. Therefore, miRNAs can be used as biomarkers for early CRCLM detection [25], particularly circulating miRNAs given their minimal invasiveness and easy accessibility. One study showed that miR-29a is significantly elevated in the serum of CRCLM patients compared to those without metastasis [26]. In fact, miR-29a can discriminate CRCLM patients from non-metastatic CRC patients with $75 \%$ sensitivity and specificity, which is more sensitive than serum CEA, with only $60 \%$ sensitivity and $34 \%$ specificity. Therefore, serum miR-29a could be a biomarker for CRCLM early detection. In a study of 224 serum samples, Yin et al[27] evaluated the expression of 11 metastasis-associated miRNAs in 116 localized CRC, 72 synchronous liver-metastatic CRC and 36 other organ-metastatic CRC. They found that the expression of serum miR-126, miR-141 and miR-21 in liver metastatic CRC were significantly different from that in localized CRC. Therefore, these three miRNAs may be used as novel biomarkers for the clinical diagnosis or prediction of early stage CRCLM. With the identification and validation of more circulating miRNA markers, early detection methods using miRNAs / miRNA signatures may be developed for clinical use in CRCLM.

\section{Therapeutic potentials of miRNAs}

It is a new strategy to treat CRCLM by targeting miRNAs, or in combination with other well-established strategies. Clinically, high expression 
of miR-29 may be predictive for the development of CRCLM. APOBEC3G, involved in CRCLM in a mouse model, was modulated by miR-29-mediated suppression of matrix metalloproteinase-2 (MMP2) to promote CRCLM [28]. Therefore, miR-29 may serve not only as a predictive marker for CRCLM, but could also be a therapeutic target. miR-622, which is overexpressed in CRC tissues and cell lines, affects metastasis and invasion of CRC cells by regulating DYRK2 and its downstream signaling pathways ${ }^{[29]}$. Nedaeinia et al. [30] transfected LS174T human colorectal adenocarcinoma cells with locked nucleic acid (LNA)-anti-miR-21 in vitro to determine the effect of miR-21 in cell growth and colony formation. At the same time, they constructed the LS174T cell chick embryo chorioallantoic membrane (CAM) module. The results showed that the LNA-anti-miR-21 in the CAM module inhibits the expression of colorectal cancer LS174T cells by regulating the PDCD4 gene, thereby reducing cell migration and liver metastasis. This provides new ideas for the development of novel miRNA therapeutics and a new direction for the treatment of CRCLM. One recent study [31] showed that an oligometastatic miR-655-3p was successfully delivered to metastasized liver using nanoscale coordination polymers (NCPs) for a targeted and prolonged distribution of miRNAs. miRNA-655-3p suppressed the tumor growth when co-delivered with oxaliplatin, suggesting additive or synergistic interactions between miRNA and platinum drugs. This was the first time the researchers systemically administered nanoparticles to deliver an oligometastatic miRNA into advanced metastatic hepatic tumors and demonstrated their tumor-suppressive effects. Their findings suggest a potential therapeutic strategy that combines tumor-suppressive miRNAs with conventional cytotoxic chemotherapies for the treatment of CRCLM. However, a single miRNA for CRCLM therapeutic target may not be effective as expected. According to Torres et al. [32], there are 38 miRNAs that were differentially expressed between highly metastatic (KM12SM/SW620) and poorly metastatic (KM12C/SW480) CRC cell lines. They found that three miRNAs (miR-424-3p, miR-503 and miR-1292) were overexpressed in both metastatic CRC cell lines and patient samples. Down-regulation of these three miRNAs shared same targets, CKB and UBA2, which increased cell adhesion and proliferation in CRC cells. This three-miRNA panel along with their two targets may constitute therapeutic biomarkers for CRCLM, suggesting that targeting multiple miRNAs may be a more efficient therapeutic strategy.

\section{Prognostic values of miRNAs}

It is suggested that miRNAs can not only assess the risks of tumor metastasis and recurrence, but also evaluate the responses of therapeutics and predict the chemo-resistance to specific drugs in CRCLM. Hur et al. [33] experimentally verified a number of miRNAs specifically associated with CRCLM in tissue and serum, which could be used to determine the prognosis. They found twenty-three miRNAs that are aberrantly expressed by comparing CRC with- and without- metastasis. Among them, four miRNAs (let-7i, miR-10b, miR-221 and miR-320a) were down-regulated in the liver metastasis group, and one (miR-885-5p) was up-regulated in the non-metastatic CRC group. It is suggested that low let-7i expression predicts possible distant metastasis and worse prognosis, whereas high miR-10b expression indicates a high incidence of distant metastasis [34]. High expression of miRNA-885-5p in serum is associated with lymph node and distant metastasis. The results were subsequently validated by RNA in situ nucleic acid hybridization. A similar study [35] revealed that miR-214 was downregulated in CRCLM, which is associated with the increased expression of FGFR-1, a target of miR-214. The data suggested that miR-214 could be used as a biomarker for poor prognosis of CRCLM patients. Lou et al. [36] found that miR-625 was significantly down-regulated in CRC tissues and cell lines, which is associated with lymph node metastasis and liver metastasis. Both in vitro and in vivo experiments demonstrated that the expression of ectopic miR-625 inhibited the invasion and metastasis of the HCT116 CRC cell line. At the same time, low miR-625 expression is associated with low survival rate, indicating that miR-625 can be used as an indicator of poor prognosis of CRC patients. miRNA-122 [37] was reported to be differentially expressed in CRCLM clinical specimens by laser capture microdissection (LCM). As a target of miR-122, cationic amino acid transporter 1 (CAT1) is expressed significantly lower in CRCLM compared to that in CRC. The analysis of 132 primary tumors revealed that the expression level of CAT1 was negatively correlated with liver metastases, and the overexpression of miRNA-122 was associated with downregulated CAT1 expression. Taken together, these data suggest that CAT1 and miR-122 could predict the prognosis of liver metastasis in CRC patients. According to some other recent studies, miR-10b [38], miR-196b-5p[39] and miR-203[40, 41] are independent risk factors for overall survival in CRC patients with liver metastases. Despite the progress being made, there is much more work that needs to be done in both animal models and clinical trials in order 
to uncover the full potential of miRNA biomarkers in CRCLM.

\section{Circulating exosomal miRNAs in CRCLM}

Exosomes are associated with cancer progression and can act in a paracrine or endocrine manner to affect cell behaviors [42]. With the progression of exosome studies in recent years, especially the discovery of the intercellular communications through exosomes and other extracellular vesicles, the potential clinical application of miRNAs and/or proteins from secreted exosomes has generated a lot of interest in the cancer research field. An exosome is a type of lipid nanoparticle with a diameter of 30-100 $\mathrm{nm}$ that are released and secreted by various living cells, containing internal proteins, mRNAs, miRNAs and other substances [43]. They can be transmitted in the circulation system and eventually absorbed by other cells. Cancer-secreted exosomes influence the tumor microenvironment and affects the growth and metastasis of cancer cells. Exosomes are a newly discovered communication medium for intercellular transporters and nucleic acid substances. They are involved in a variety of cellular processes, such as intercellular communication, cell migration, angiogenesis and immune regulation. Compared to direct cell contact or secretory factors, exosomes are more targeted and can affect the function of recipient cells more efficiently. They play an important role in cancer, infectious diseases and neurodegenerative diseases. Currently, there are no standard agency-approved, exosome-based diagnostic, therapeutic, or prognostic clinical tests for CRCLM. miRNAs contained in exosomes derived from CRC cells are closely related to oncogenes and tumor metastasis-related genes, suggesting that they may be related to CRCLM, and play a role in the initiation and development of CRCLM [44].

Compared to the general population, cancer patients have larger numbers of circulating exosomes [45]. Therefore, circulating exosomal miRNAs may be used for diagnosis or prognosis for CRC patients. Reliable blood test could be a perfect companion tool for routine imaging examination of CRCLM patients or for early detection of CRCLM. Exosomal miRNAs are considered the main source of circulating miRNAs isolated from serum or plasma [46]. Currently, there are a few studies showing the analysis of circulating exosomal miRNAs in early diagnosis of CRCLM. Matsumura et al. [44] analyzed the expression of exosomal miRNAs in 227 serum samples from CRC patients. Compared with patients without recurrence, expression of 18 exosomal miRNAs was increased while 46 exosomal miRNAs decreased. Among them, six exosomal miRNAs (miR-19a, miR-19b, miR-23a,
miR-92a, miR-320a and miR-4437) were associated with the development of liver metastasis, rendering them to be potential biomarkers for CRCLM early detection. Further studies suggested that the CRC patients with high level of exosomal miR-19a expression were associated with poor prognosis compared to the low expression group. This may indicate that the exosomal miR-19a could be a potential marker of prognosis in CRCLM.

At present, there are a few studies that focus on the clinical applications of circulating exosomal miRNAs in CRCLM. The stability and reproducibility of exosomal miRNA detection and its independent features require more research to be evaluated and validated. Hence, the potential clinical impact on CRCLM cannot be ignored. As the research technology matures and deepens, the detection of circulating exosomal miRNAs may become a very promising field for identifying CRCLM biomarkers.

In recent studies, the functional studies in cancer cell lines or xenograft models have revealed the mechanisms of exosomal miRNAs in cancer cells along with another cell type of TME in supporting or inhibiting tumor growth, transferring drug resistance, and preparing for the metastatic niche [47].

Based on the study by $\mathrm{Fu}$ et al. [48], there are 25 miRNAs being up-regulated and 5 miRNAs being down-regulated in exosomes purified from SW620 culture supernatant. Candidate miRNAs were further evaluated for CRC diagnosis by using qRT-PCR. High expression levels of circulating exosomal miR-17-5p and miR-92a-3p were associated with the stages of CRC patients, indicating that these exosomal miRNAs could be biomarkers for CRC and / or CRCLM. The data further showed that exosomal miR-7, miR-181a-5p, miR-192, miR-194, and miR-375, which are related to multiple biofunctions such as inflammation, fibrosis, and angiogenesis, may also play an important role in CRCLM.

Valcz et al. ${ }^{[49]}$ detected the expression of the Alix protein, which are stably present in exosomes, from normal intestinal epithelial tissue, adenoma tissue and colorectal cancer tissue. They discovered that exosomes play an important role in promoting the carcinogenesis of colonic epithelium and migration. Recent studies show that the expression of exosomal miR-210 was significantly higher compared with its intracellular levels in adherently growing HCT-8 cells, and correlated to anoikis resistance and EMT markers. Exosomes can induce cancerous growth of normal epithelial cells by promoting EMT through miRNA-210 [50] and can affect the adhesion and migration of CRC cells.

Teng et al. [51] examined different exosomal miRNA expression profiles in primary mouse colon 
tumor, CRCLM and naïve colon tissues. They found that exosomal miR-193a interacts with major vault protein (MVP), causing cell cycle G1 arrest and cell proliferation repression by targeting Caprin1. This suggests that MVP-mediated selective sorting of tumor suppressor miRNA into exosomes promotes tumor progression and liver migration.

We are still in the early stages of exosomal research in CRCLM. Since exosomes and exosomal miRNAs can be detected from patients non-invasively through blood, urine or other body fluids, these microvesicles could be potential sources of diagnostic, prognostic or predictive biomarkers of CRCLM. It is likely that the studies focusing on circulating exosomal miRNAs analysis in patient blood, urine or other body as liquid biopsy research are a step forward towards precision medicine.

\section{Discussion and conclusions}

Liver metastasis is an important biological feature of CRC, and it is also an important factor of recurrence and poor prognosis of CRC patients. Combining molecular and traditional treatments may lead to an improved Overall Survival (OS), a reduction in intrahepatic recurrence, and a decreased toxicity of perioperative therapies. The process of liver metastasis of CRC depends on multiple complex interactions between cancer cells in the tumor and host-derived cells in the microenvironment in both the primary tumor and secondary organs. In contrast to the complex and diverse oncogenes and tumor suppressor genes involved in cancer, miRNAs with tumor promoting or tumor suppressing effects have many more advantages [52]. Identification of miRNAs, including exosomal miRNAs, involved in liver metastasis could lead to the development of more accurate detection methods for early diagnosis, treatment and prognosis of CRCLM. With the continued improvements of miRNA expression profiling, and the development of more stable, effective and low-toxic miRNA mimics and inhibitors, the number of drugs targeting miRNAs has increased at much faster pace. It seems that miRNA-based therapeutics as adjuvant tools of targets will be realized in the near future once we overcome the technical limitations. Although the research on miRNAs especially the exosomal miRNAs in CRCLM are full of challenges, the prospects are still very broad.

\section{Acknowledgements}

We thank Mr. Davis Tran for his critical reading and edits for the manuscript. This research was supported by the Elaine H. Snyder Cancer Research Award (to SWF).

\section{Conflicts of Interest}

The authors declare that they have no competing interests.

\section{References}

1. Strubberg AM, Madison BB: MicroRNAs in the etiology of colorectal cancer: pathways and clinical implications. Dis Model Mech 2017, 10(3):197-214.

2. Siegel RL, Miller KD, Jemal A: Cancer statistics, 2018. CA Cancer J Clin 2018, 68(1):7-30.

3. Tomlinson JS, Jarnagin WR, DeMatteo RP, Fong Y, Kornprat P, Gonen M, Kemeny N, Brennan MF, Blumgart LH, D'Angelica M: Actual 10-year survival after resection of colorectal liver metastases defines cure. I Clin Oncol 2007, 25(29):4575-4580.

4. House MG, Kemeny NE, Gonen M, Fong Y, Allen PJ, Paty PB, DeMatteo RP, Blumgart LH, Jarnagin WR, D'Angelica MI: Comparison of adjuvant systemic chemotherapy with or without hepatic arterial infusional chemotherapy after hepatic resection for metastatic colorectal cancer. Ann Surg 2011, 254(6):851-856.

5. Misiakos EP, Karidis NP, Kouraklis G: Current treatment for colorectal liver metastases. World J Gastroenterol 2011, 17(36):4067-4075.

6. Zarour LR, Anand S, Billingsley KG, Bisson WH, Cercek A, Clarke MF, Coussens LM, Gast CE, Geltzeiler CB, Hansen L et al: Colorectal Cancer Liver Metastasis: Evolving Paradigms and Future Directions. Cell Mol Gastroenterol Hepatol 2017, 3(2):163-173.

7. Schetter AJ, Okayama H, Harris CC: The role of microRNAs in colorectal cancer. Cancer I 2012, 18(3):244-252.

8. Wang D, Liu J, Huo T, Tian Y, Zhao L: The role of microRNAs in colorectal liver metastasis: Important participants and potential clinical significances. Tumour Biol 2017, 39(6):1010428317709640.

9. Bracken CP, Scott HS, Goodall GJ: A network-biology perspective of microRNA function and dysfunction in cancer. Nat Rev Genet 2016, 17(12):719-732.

10. Lin M, Chen W, Huang J, Gao H, Ye Y, Song Z, Shen X: MicroRNA expression profiles in human colorectal cancers with liver metastases. Oncol Rep 2011, 25(3):739-747.

11. Peng Y, Croce CM: The role of MicroRNAs in human cancer. Signal Transduct Target Ther 2016, 1:15004.

12. Cellura D, Pickard K, Quaratino S, Parker H, Strefford JC, Thomas GJ, Mitter $\mathrm{R}$, Mirnezami AH, Peake NJ: miR-19-Mediated Inhibition of Transglutaminase-2 Leads to Enhanced Invasion and Metastasis in Colorectal Cancer. Mol Cancer Res 2015, 13(7):1095-1105.

13. Lam CSC, Ng L, Chow AKM, Wan TMH, Yau S, Cheng NSM, Wong SKM, Man JHW, Lo OSH, Foo DCC et al: Identification of microRNA 885-5p as a novel regulator of tumor metastasis by targeting CPEB2 in colorectal cancer. Oncotarget 2017, 8(16):26858-26870.

14. Cheng $\mathrm{D}$, Zhao $\mathrm{S}$, Tang $\mathrm{H}$, Zhang $\mathrm{D}$, Sun $\mathrm{H}$, Yu F, Jiang $\mathrm{W}$, Yue B, Wang J, Zhang $\mathrm{M}$ et al: MicroRNA-20a-5p promotes colorectal cancer invasion and metastasis by downregulating Smad4. Oncotarget 2016, 7(29):45199-45213.

15. Yan LX, Huang XF, Shao Q, Huang MY, Deng L, Wu QL, Zeng YX, Shao JY: MicroRNA miR-21 overexpression in human breast cancer is associated with advanced clinical stage, lymph node metastasis and patient poor prognosis. Rna 2008, 14(11):2348-2360.

16. Shibuya $\mathrm{H}$, Iinuma $\mathrm{H}$ Shimada $\mathrm{R}$ Horiuchi $\mathrm{A}$ Watanabe $\mathrm{T}$ : Clinicopathological and prognostic value of microRNA-21 and microRNA-155 in colorectal cancer. Oncology 2010, 79(3-4):313-320.

17. Ji D, Chen Z, Li M, Zhan T, Yao Y, Zhang Z, Xi J, Yan L, Gu J: MicroRNA-181a promotes tumor growth and liver metastasis in colorectal cancer by targeting the tumor suppressor WIF-1. Mol Cancer 2014, 13:86.

18. Li J, Du L, Yang Y, Wang C, Liu H, Wang L, Zhang X, Li W, Zheng G, Dong Z: MiR-429 is an independent prognostic factor in colorectal cancer and exerts its anti-apoptotic function by targeting SOX2. Cancer Lett 2013, 329(1):84-90.

19. Chen J, Wang Y, Zhuo L, Liu Z, Liu T, Li W, Cai Y, Zheng H: Fas signaling induces stemness properties in colorectal cancer by regulation of Bmi1. Mol Carcinog 2017, 56(10):2267-2278.

20. Li Y, Sun Z, Liu B, Shan Y, Zhao L, Jia L: Tumor-suppressive miR-26a and miR-26b inhibit cell aggressiveness by regulating FUT4 in colorectal cancer. Cell Death Dis 2017, 8(6):e2892.

21. Laudato S, Patil N, Abba ML, Leupold JH, Benner A, Gaiser T, Marx A, Allgayer H: P53-induced miR-30e-5p inhibits colorectal cancer invasion and metastasis by targeting ITGA6 and ITGB1. Int I Cancer 2017, 141(9):1879-1890.

22. Pecqueux M, Liebetrau I, Werft W, Dienemann H, Muley T, Pfannschmidt J, Mussle B, Rahbari N, Scholch S, Buchler MW et al: A Comprehensive MicroRNA Expression Profile of Liver and Lung Metastases of Colorectal Cancer with Their Corresponding Host Tissue and Its Prognostic Impact on Survival. Int J Mol Sci 2016, 17(10).

23. Li J, Chen Y, Guo X, Zhou L, Jia Z, Tang Y, Lin L, Liu W, Ren C: Inhibition of miR-15b decreases cell migration and metastasis in colorectal cancer. Tumour Biol 2016, 37(7):8765-8773.

24. Park YR, Kim SL, Lee MR, Seo SY, Lee JH, Kim SH, Kim IH, Lee SO, Lee ST, Kim SW: MicroRNA-30a-5p (miR-30a) regulates cell motility and EMT by 
directly targeting oncogenic TM4SF1 in colorectal cancer. J Cancer Res Clin Oncol 2017, 143(10):1915-1927.

25. Manne U, Jadhav T, Putcha BK, Samuel T, Soni S, Shanmugam C, Suswam EA: Molecular Biomarkers of Colorectal Cancer and Cancer Disparities: Current Status and Perspective. Curr Colorectal Cancer Rep 2016, 12(6):332-344.

26. Wang LG, Gu J: Serum microRNA-29a is a promising novel marker for early detection of colorectal liver metastasis. Cancer Epidemiol 2012, 36(1):e61-67.

27. Yin J, Bai Z, Song J, Yang Y, Wang J, Han W, Zhang J, Meng H, Ma X, Yang Y et al: Differential expression of serum miR-126, miR-141 and miR-21 as novel biomarkers for early detection of liver metastasis in colorectal cancer. Chin J Cancer Res 2014, 26(1):95-103.

28. Ding Q, Chang CJ, Xie X, Xia W, Yang JY, Wang SC, Wang Y, Xia J, Chen L, Cai Cet al: APOBEC3G promotes liver metastasis in an orthotopic mouse model of colorectal cancer and predicts human hepatic metastasis. J Clin Invest 2011, 121(11):4526-4536.

29. Wang Y, Sun J, Wei X, Luan L, Zeng X, Wang C, Zhao W: Decrease of miR-622 expression suppresses migration and invasion by targeting regulation of DYRK2 in colorectal cancer cells. Onco Targets Ther 2017, 10:1091-1100.

30. Nedaeinia R, Sharifi M, Avan A, Kazemi M, Nabinejad A, Ferns GA, Ghayour-Mobarhan M, Salehi R: Inhibition of microRNA-21 via locked nucleic acid-anti-miR suppressed metastatic features of colorectal cancer cells through modulation of programmed cell death 4. Tumour Biol 2017, 39(3):1010428317692261.

31. Oshima G, Guo N, He C, Stack ME, Poon C, Uppal A, Wightman SC, Parekh A, Skowron KB, Posner MC et al: In Vivo Delivery and Therapeutic Effects of a MicroRNA on Colorectal Liver Metastases. Mol Ther 2017, 25(7):1588-1595.

32. Torres S, Garcia-Palmero I, Bartolome RA, Fernandez-Acenero MJ, Molina E, Calvino E, Segura MF, Casal JI: Combined miRNA profiling and proteomics demonstrates that different miRNAs target a common set of proteins to promote colorectal cancer metastasis. J Pathol 2017, 242(1):39-51.

33. Hur K, Toiyama Y, Schetter AJ, Okugawa Y, Harris CC, Boland CR, Goel A: Identification of a metastasis-specific MicroRNA signature in human colorectal cancer. J Natl Cancer Inst 2015, 107(3).

34. Ma L, Teruya-Feldstein J, Weinberg RA: Tumour invasion and metastasis initiated by microRNA-10b in breast cancer. Nature 2007, 449(7163):682-688.

35. Chen DL, Wang ZQ, Zeng ZL, Wu WJ, Zhang DS, Luo HY, Wang F, Qiu MZ, Wang DS, Ren Cet al: Identification of microRNA-214 as a negative regulator of colorectal cancer liver metastasis by way of regulation of fibroblast growth factor receptor 1 expression. Hepatology 2014, 60(2):598-609.

36. Lou X, Qi X, Zhang Y, Long H, Yang J: Decreased expression of microRNA-625 is associated with tumor metastasis and poor prognosis in patients with colorectal cancer. I Surg Oncol 2013, 108(4):230-235.

37. Iino I, Kikuchi H, Miyazaki S, Hiramatsu Y, Ohta M, Kamiya K, Kusama Y, Baba S, Setou M, Konno H: Effect of miR-122 and its target gene cationic amino acid transporter 1 on colorectal liver metastasis. Cancer Sci 2013, 104(5):624-630

38. Nishida N, Yamashita S, Mimori K, Sudo T, Tanaka F, Shibata K, Yamamoto $\mathrm{H}$, Ishii $\mathrm{H}$, Doki $\mathrm{Y}$, Mori M: MicroRNA-10b is a Prognostic Indicator in Colorectal Cancer and Confers Resistance to the Chemotherapeutic Agent 5-Fluorouracil in Colorectal Cancer Cells. Ann Surg Oncol 2012, 19(9):3065-3071

39. Stiegelbauer V, Vychytilova-Faltejskova P, Karbiener M, Pehserl AM, Reicher A, Resel M, Heitzer E, Ivan C, Bullock M, Ling H et al: miR-196b-5p Regulates Colorectal Cancer Cell Migration and Metastases through Interaction with HOXB7 and GALNT5. Clin Cancer Res 2017, 23(17):5255-5266.

40. Li WH, Chang JJ, Tong D, Peng JJ, Huang D, Guo WJ, Zhang W, Li J: Differential microRNA expression profiling in primary tumors and matched liver metastasis of patients with colorectal cancer. Oncotarget 2017, 8(22):35783-35791

41. Hur K, Toiyama Y, Okugawa Y, Ide S, Imaoka H, Boland CR, Goel A: Circulating microRNA-203 predicts prognosis and metastasis in human colorectal cancer. Gut 2017, 66(4):654-665.

42. Carvalho J, Oliveira C: Extracellular Vesicles - Powerful Markers of Cancer EVolution. Front Immunol 2014, 5:685.

43. Ruiz-Lopez L, Blancas I, Garrido JM, Mut-Salud N, Moya-Jodar M, Osuna A, Rodriguez-Serrano F: The role of exosomes on colorectal cancer: A review. I Gastroenterol Hepatol 2018, 33(4):792-799.

44. Matsumura T, Sugimachi K, Iinuma H, Takahashi Y, Kurashige J, Sawada G, Ueda M, Uchi R, Ueo $\mathrm{H}$, Takano $Y$ et al: Exosomal microRNA in serum is a novel biomarker of recurrence in human colorectal cancer. Br J Cancer 2015, 113(2):275-281

45. Kalluri R: The biology and function of exosomes in cancer. J Clin Invest 2016, 126(4):1208-1215.

46. Nedaeinia R, Manian M, Jazayeri MH, Ranjbar M, Salehi R, Sharifi M, Mohaghegh F, Goli M, Jahednia SH, Avan A et al: Circulating exosomes and exosomal microRNAs as biomarkers in gastrointestinal cancer. Cancer Gene Ther 2017, 24(2):48-56.

47. Pencheva N, Tavazoie SF: Control of metastatic progression by microRNA regulatory networks. Nat Cell Biol 2013, 15(6):546-554.

48. Fu F, Jiang $\mathrm{W}$, Zhou L, Chen Z: Circulating Exosomal miR-17-5p and miR-92a-3p Predict Pathologic Stage and Grade of Colorectal Cancer. Transl Oncol 2018, 11(2):221-232.

49. Valcz G, Galamb O, Krenacs T, Spisak S, Kalmar A, Patai AV, Wichmann B, Dede K, Tulassay Z, Molnar B: Exosomes in colorectal carcinoma formation: ALIX under the magnifying glass. Mod Pathol 2016, 29(8):928-938.
50. Bigagli E, Luceri C, Guasti D, Cinci L: Exosomes secreted from human colon cancer cells influence the adhesion of neighboring metastatic cells: Role of microRNA-210. Cancer Biol Ther 2016:1-8.

51. Teng Y, Ren Y, Hu X, Mu J, Samykutty A, Zhuang X, Deng Z, Kumar A, Zhang L, Merchant ML et al: MVP-mediated exosomal sorting of miR-193a promotes colon cancer progression. Nat Commun 2017, 8:14448.

52. Dong $\mathrm{Y}, \mathrm{Wu} \mathrm{WK}, \mathrm{Wu} \mathrm{CW}$, Sung JJ, Yu J, Ng SS: MicroRNA dysregulation in colorectal cancer: a clinical perspective. Br J Cancer 2011, 104(6):893-898. 